\title{
Nanoelectronic Device Structures at Terahertz Frequency
}

\begin{abstract}
M. Horák
Potential barriers of different types (rectangular, triangle, parabolic) with a dc-bias and a small ac-signal in the THz-frequency band are investigated in this paper. The height of the potential barrier is modulated by the high frequency signal. If electrons penetrate through the barrier they can emit or absorb usually one or even more energy quanta $\hbar \omega$, thus the electron wave function behind the barrier is a superposition of different harmonics $\exp (-i n \omega t)$. The time-dependent Schrödinger equation is solved to obtain the reflection and transmission amplitudes and the barrier transmittance corresponding to the harmonics. The electronic current density is calculated according to the Tsu-Esaki formula. If the harmonics of the electron current density are known, the complex admittance and other electrical parameters of the structure can be found.
\end{abstract}

Keywords: Nanoelectronic, terahertz, potential barrier, transmittance, Schrödinger equation.

\section{Introduction}

The terahertz frequency band is recently usually considered as the interval $300 \mathrm{GHz}-3 \mathrm{THz}$ that corresponds to the submillimeter wavelength range between $1 \mathrm{~mm}$ and $100 \mu \mathrm{m}$ or to photon energy within the range $1.2-12.4 \mathrm{meV}$. Despite great scientific interest the terahertz frequency range remains one of the least tapped regions of the electromagnetic spectrum. Below $300 \mathrm{GHz}$ we cross into the millimeter-wave bands. Beyond $3 \mathrm{THz}$ is more or less unclaimed territory: the border between far-infrared and submillimeter radiation is still rather blurry.

Recent rapid progress in nanoelectronics and high frequency technologies necessitates that heterojunctions, superlattices, low-dimensional semiconductor structures, quantum wells and barriers are today standard building blocks of modern electronic devices, which find their application in the field of microwave and submillimeter technology or in photonics. The existence of quantum wells and barriers results in the quantum-based mechanism of electron transport, thermionic emission across the barrier and the tunnelling (thermionic-field-emission) through the barrier. These effects should be treated by means of appropriate methods of quantum physics.

Although the frequency of $1 \mathrm{THz}$ appears to be very high, this is only an appearance. The frequency $1.8 \mathrm{GHz}$ is at present in general use in mobile telephones. It is clear that $1.8 \mathrm{GHz}$ cannot be equal to the transient frequency $f_{\mathrm{T}}$ of transistors in the integrated circuits of mobile telephones. The frequency $1.8 \mathrm{GHz}$ should be even lower than the frequency $f_{\beta}$, which is defined by the $3 \mathrm{~dB}$ drop of the current gain this means that the frequency $f_{\mathrm{T}}$ should be of the order $(100 \div 300)$ GHz. Even higher frequency bands are used in radar systems. The resonant tunneling diode (and similar structures with resonant tunneling) are recently typical devices for the terahertz fequency band [1]. Moreover, nearly the same theoretical approach that is used for investigating of the terahertz frequency band can also be applied if the interaction of near-infrared radiation with photonic structures is studied. Quantum cascade lasers may provide terahertz bandwith for communications [2].

A typical situation in electronics is that a dc-bias together with a small ac-signal are applied to the structure. The calcu- lation of potential barrier transmittance with dc-bias only is a classical and well-known problem of quantum mechanics [3]. The application of a high-frequency signal to the barrier has been studied only in the last decade. A general method of solution is described in $[4,5]$ and developed in $[6,7]$. However, papers concerning high-frequency phenomena are usually devoted to resonant tunnelling diodes (RTD) and different types of potential barriers are rarely investigated, e.g. in [8] (the high-frequency potential step with zero steady state potential). The aim of this paper is to present results achieved in a theoretical investigation of the high-frequency electron transport across the rectangular, triangular, trapezoidal or parabolic potential barriers that are most frequently used in various nanoelectronic or photonic structures.

\section{Steady-state transmittance of a potential barrier}

The term "steady-state" means that only $d c$-bias is applied to the potential barrier. Although we have mentioned above that the calculation of steady-state barrier transmittance is a classical problem of quantum physics, we briefly summarise and generalise these results.

We will consider a rectangular, triangular, trapezoidal or parabolic potential barrier, see Fig. 1. We assume that there is a significant voltage drop only at the barrier region, i.e. outside the voltage barrier there is no electric field and the electrons can be described as free. The barrier height $U_{\max }$, the barrier width $x_{\mathrm{B}}$, and in fact the whole barrier profile, i.e. the potential energy $U(x)$, depend on the external applied bias. The formulae for the potential energy $U(x)$ are given in Fig. 1 .

Consider that the electrons are incident on the barrier from the left. In this case, in region A we have incident electrons described by the wave function $e^{i k_{0} x}\left(k_{0}\right.$ is the wave vector related to the electron kinetic energy by $E=\hbar^{2} k_{0}^{2} / 2 m$ ) and electrons reflected from the barrier with wave function $r_{0} e^{-i k_{0} x}\left(r_{0}\right.$ is the reflection amplitude). The electrons inside the barrier region $\mathrm{B}$ are described by some specific wave function that is dependent on the profile (shape) of the potential barrier. In region $\mathrm{C}$ (after the barrier) are transmitted only electrons with wave function $t_{0} e^{i q_{0} x}\left(t_{0}\right.$ is the transmission amplitude) with the wave vector $q_{0}$ satisfying 


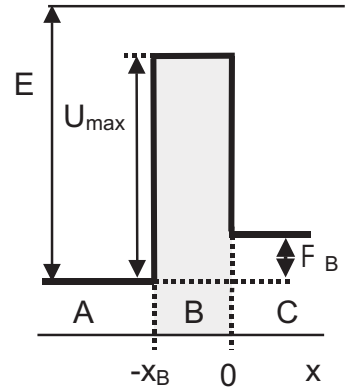

rectangular barrier

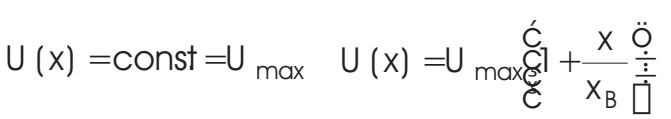
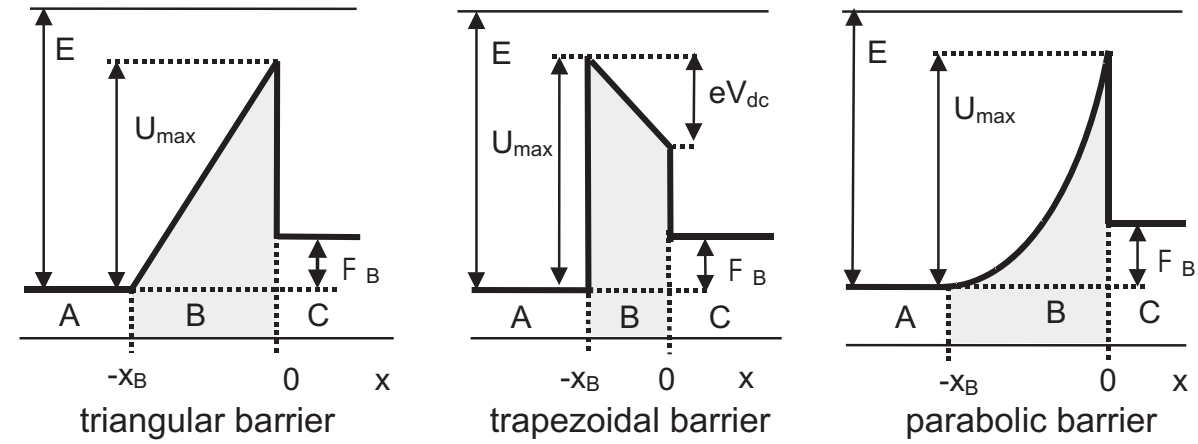

$U(x)=U_{\text {max }}-e V_{d c} \underset{c}{\stackrel{c}{c}}+\frac{x}{x_{B}} \frac{\ddot{\dot{i}}}{\dot{\dot{\square}}}$

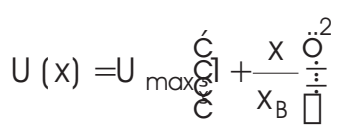

Fig. 1: Different types of potential barrier; $E$ is the energy of the incident electron, $U(x)$ is the potential energy in the barrier region B

$E+\Phi_{\mathrm{B}}=\hbar^{2} q_{0}^{2} / 2 m$. Thus the electron wave functions in regions A, B, C are:

$$
\begin{aligned}
& \varphi_{\mathrm{A}}(x)=e^{i k_{0} x}+r_{0} e^{-i k_{0} x}, \\
& \varphi_{\mathrm{B}}(x)=a_{0} f(x)+b_{0} g(x), \\
& \varphi_{\mathrm{C}}(x)=t_{0} e^{i q_{0} x} .
\end{aligned}
$$

Let us turn our attention to the functions $f(x), g(x)$ in Eq. (1). These functions contain the information on the potential barrier. In general the wave function $\varphi_{\mathrm{B}}(x)$ inside the barrier region is the eigenfunction of the corresponding hamiltonian, i.e. it is the solution of the stationary Schrödinger equation

$\mathrm{H}_{\mathrm{dc}} \varphi_{\mathrm{B}}=E \varphi_{\mathrm{B}} \quad$ where $\mathrm{H}_{\mathrm{dc}}=-\frac{\hbar^{2}}{2 m} \frac{d^{2}}{d x^{2}}+U(x)$

For a rectangular barrier we obtain

$$
f(x)=e^{i p_{0} x}, \quad g(x)=e^{-i p_{0} x}, \quad p_{0}=\sqrt{2 m\left(E-U_{\max }\right)} / \hbar
$$

where $p_{0}$ is real for $E>U_{\max }$ (this corresponds to the electron emission over the barrier) and $p_{0}$ is imaginary, $p_{0}=i \kappa_{0}$ for $E<U_{\max }$ (electron tunneling through the barrier). The results for the potential barriers in Fig. 1 are summarized in Table 1.

The electron wave functions for any type of barrier obey the standard boundary conditions at the interfaces $x=-x_{B}$, $x=0$ (to simplify the problem equal electron effective mass $m$ is considered throughout the structure):

$\varphi_{\mathrm{A}}\left(-x_{\mathrm{B}}\right)=\varphi_{\mathrm{B}}\left(-x_{\mathrm{B}}\right), \quad \varphi_{\mathrm{A}}^{\prime}\left(-x_{\mathrm{B}}\right)=\varphi_{\mathrm{B}}^{\prime}\left(-x_{\mathrm{B}}\right)$,

$\varphi_{\mathrm{B}}(0)=\varphi_{\mathrm{C}}(0), \quad \varphi_{\mathrm{B}}^{\prime}(0)=\varphi_{\mathrm{C}}^{\prime}(0)$.

Substituting the wave functions we obtain a system of four linear equations for the unknown coefficients $r_{0}, t_{0}, a_{0}, b_{0}$ in (1). As the system is sufficiently simple, it can be solved analytically. If the wave functions are known, the single electron

\begin{tabular}{|c|c|c|}
\hline $\begin{array}{l}\text { potential barrier } \\
\text { (see Fig. 1) }\end{array}$ & $\begin{array}{l}\text { functions } f(x), g(x) \\
\quad \text { (see Eq. 1) }\end{array}$ & parameters \\
\hline rectangular & $f(x)=e^{i p_{0} x}, g(x)=e^{-i p_{0} x}$ & $p_{0}=\sqrt{2 m\left(E-U_{\max }\right)} / \hbar$ \\
\hline triangular & $\begin{array}{c}f(x) \mapsto A i(\xi), g(x) \mapsto B i(\xi) \\
\text { Airy functions }\end{array}$ & $\xi=\beta\left[x+x_{\mathrm{B}}\left(1-\frac{E}{U_{\max }}\right)\right], \quad \beta=\left[\frac{2 m U_{\max }}{\hbar^{2} x_{\mathrm{B}}}\right]^{1 / 3}$ \\
\hline trapezoidal & $\begin{array}{c}f(x) \mapsto A i(\eta), g(x) \mapsto B i(\eta) \\
\text { Airy functions }\end{array}$ & $\begin{array}{l}\eta=-\gamma(x-\lambda) \\
\gamma=\left(\frac{2 m\left(U_{\max }+\Phi_{\mathrm{B}}\right)}{\hbar^{2} x_{\mathrm{B}}}\right)^{1 / 3}, \lambda=-x_{\mathrm{B}} \frac{e-\Phi_{\mathrm{B}}}{U_{\max }+\Phi_{\mathrm{B}}}\end{array}$ \\
\hline parabolic & $\begin{array}{c}f(x) \mapsto U(-u, \zeta), g(x) \mapsto V(-u, \zeta) \\
\text { parabolic cylinder functions }\end{array}$ & $\begin{array}{l}\zeta=\alpha\left(x+x_{\mathrm{B}}\right) \\
\alpha=\left(\frac{8 m U_{\max }}{\hbar^{2} x_{\mathrm{B}}^{2}}\right)^{1 / 4}, u=E \frac{x_{\mathrm{B}}}{\hbar}\left(\frac{m}{2 U_{\max }}\right)^{1 / 2}\end{array}$ \\
\hline
\end{tabular}
quantum mechanical current densities of incident and transmitted electrons can be calculate according to the well-known formulae

Table 1: Electron wave functions in the barrier region 


$$
\begin{aligned}
& j_{i n c}=\frac{e \hbar}{2 i m}\left(\varphi_{\mathrm{A}}^{*} \frac{\partial \varphi_{\mathrm{A}}}{\partial x}-\varphi_{\mathrm{A}} \frac{\partial \varphi_{\mathrm{A}}^{*}}{\partial x}\right), \\
& j_{\text {trans }}=\frac{e \hbar}{2 i m}\left(\varphi_{\mathrm{C}}^{*} \frac{\partial \varphi_{\mathrm{C}}}{\partial x}-\varphi_{\mathrm{C}} \frac{\partial \varphi_{\mathrm{C}}^{*}}{\partial x}\right) .
\end{aligned}
$$

The steady state barrier transmittance $T_{\mathrm{dc}}(E)$ is a function of electron energy $E$ and it is defined as the ratio $\left|j_{\text {trans }} / j_{\text {inc }}\right|$. We introduce the following short notation:

$$
\begin{array}{ll}
A=f(x=0), & B=g(x=0), \\
A^{\prime}=f^{\prime}(x=0), & B^{\prime}=g^{\prime}(x=0), \\
C=f\left(x=x_{\mathrm{B}}\right), & D=g\left(x=x_{\mathrm{B}}\right), \\
C^{\prime}=f^{\prime}\left(x=x_{\mathrm{B}}\right), & D^{\prime}=g^{\prime}\left(x=x_{\mathrm{B}}\right) \\
\gamma_{1}=\frac{\Gamma}{k}, \quad \gamma_{2}=\frac{q}{\Gamma}, & \Gamma=q_{0} / \beta / \gamma / \alpha
\end{array}
$$

for the

rectangular / triangular / trapeziodal / parabolic barrier by

The transmission amplitude $t_{0}$ defined in $(1)$ is then given $t_{0}=\frac{2}{\pi} e^{-i k x_{\mathrm{B}}}\left[\left(B^{\prime}-i \gamma_{2} B\right)\left(C-i \gamma_{1} C^{\prime}\right)-\left(A^{\prime}-i \gamma_{2} A\right)\left(D-i \gamma_{1} D^{\prime}\right)\right]^{-1}$

and the transmittance reads

$$
T_{\mathrm{dc}}(E)=\frac{q_{0}}{k_{0}}\left|t_{0}\right|^{2}
$$

Let us consider the $\mathrm{N}-\mathrm{Al}_{1-x} \mathrm{Ga}_{x} \mathrm{As} / \mathrm{p}^{+}$-GaAs abrupt heterojunction with the following parameters: aluminium mole fraction 0.35 , donor concentration in N-region $5 \times 10^{17} \mathrm{~cm}^{-3}$, acceptor concentration in $\mathrm{p}^{+}$-region $1 \times 10^{19} \mathrm{~cm}^{-3}$, the depletion layer extends in the N-region and its width is $x_{\mathrm{n}} \approx 65 \mathrm{~nm}$ for zero bias, the heterojunction built-in voltage is $V_{\mathrm{bi}}=1.8 \mathrm{~V}$. The electron effective mass is considered to be the same throughout the structure and equal to the effective mass of an electron in GaAs, thus $m=0.067 \mathrm{~m}_{\mathrm{el}}$. The energy $U_{\text {max }}$ is related to the built-in voltage $V_{\mathrm{bi}}$ and to the external applied voltage as $U_{\max }=e\left(V_{\mathrm{bi}}-V_{a}\right)$. The conduction band profile for various forward bias and the barrier transmittance are shown in Fig. 2.

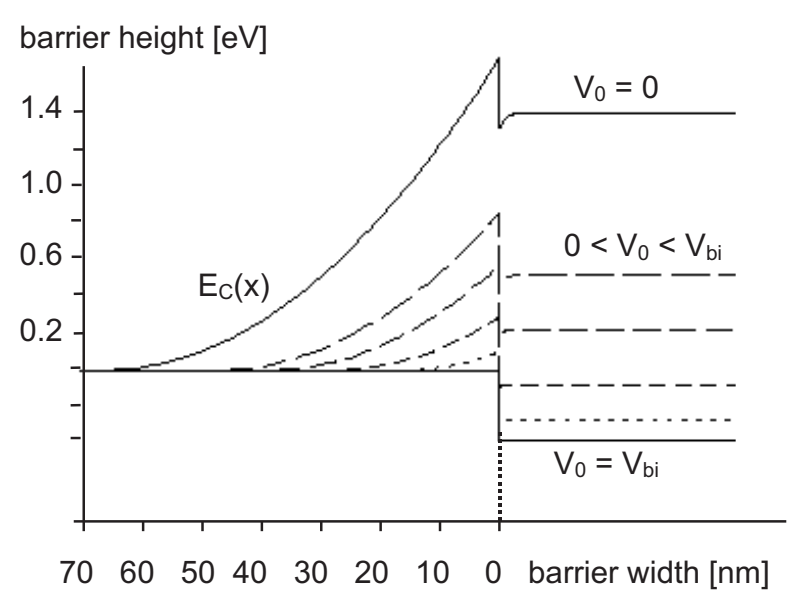

\section{Electron wave function in barrier region with high frequency modulation}

We will now consider the case if the potential barrier is modulated by a high frequency signal $V_{\mathrm{ac}} \cos (\omega t)$ where the angular frequency $w \approx(0.1 \div 10)$ THz and the amplitude $V_{\mathrm{ac}}$ is small and constant; such modulation is called homogeneous. The more general and more complicated case of non-homogeneous modulation $V_{\mathrm{ac}}(x) \cos (\omega t)$ is not considered in this paper. The electron wave function $\psi_{\mathrm{B}}(x, t)$ inside the barrier region is the solution of the time-dependent Schrödinger equation with the hamiltonian $\mathrm{H}_{\mathrm{dc}}+\mathrm{H}_{\mathrm{ac}}$ where $\mathrm{H}_{\mathrm{dc}}$ according to (2) represents the barrier profile (including the dc bias) and $\mathrm{H}_{\mathrm{ac}}$ stands for the high frequency modulation

$$
\begin{aligned}
& i \hbar \frac{\partial \psi_{\mathrm{B}}}{\partial t}=\left(\mathrm{H}_{\mathrm{dc}}+\mathrm{H}_{\mathrm{ac}}\right) \psi_{\mathrm{B}}, \\
& \mathrm{H}_{\mathrm{dc}}=-\frac{\hbar^{2}}{2 m} \frac{\partial^{2}}{\partial x^{2}}+U(x), \\
& \mathrm{H}_{\mathrm{ac}}=e V_{\mathrm{ac}} \cos (\omega t)
\end{aligned}
$$

It can be immediately proved that the wave function is

$\psi_{\mathrm{B}}(x, t)=\exp \left(-i \frac{E}{\hbar} t\right) \exp \left(-i \frac{e V_{\mathrm{ac}}}{\hbar \omega} \sin (\omega t)\right) \varphi_{\mathrm{B}}(x)$

where $\varphi_{\mathrm{B}}(x)$ is the solution of the stationary Schrödinger equation (2). We can see that the problem of describing electron wave functions in a uniform sinusoidally oscillating potential (9) is identical to the problem of frequency modulation in telecommunications or in signal theory. The wave function (10) can be considered as the frequency modulated wave with carrier frequency $w_{0}=E / \hbar$, see Fig. 3 .

We apply the Bessel function expansion to the second term of (10)

$\exp \left(-i \frac{e V_{\mathrm{ac}}}{\hbar \omega} \sin (\omega t)\right)=\sum_{p=-\infty}^{+\infty} J_{p}\left(\frac{e V_{\mathrm{ac}}}{\hbar \omega}\right) \exp (-i p \omega t)$.

This expansion enables us to consider the wave function (10) as the superposition of harmonics $\exp (-i p \omega t)$, $p=0, \pm 1, \pm 2, \ldots$, see Fig. 4 . Thus, passing the barrier region, the electron is able to absorb or emit one or more energy

\section{barrier}

transmittance $\mathrm{T}_{\mathrm{dc}}$

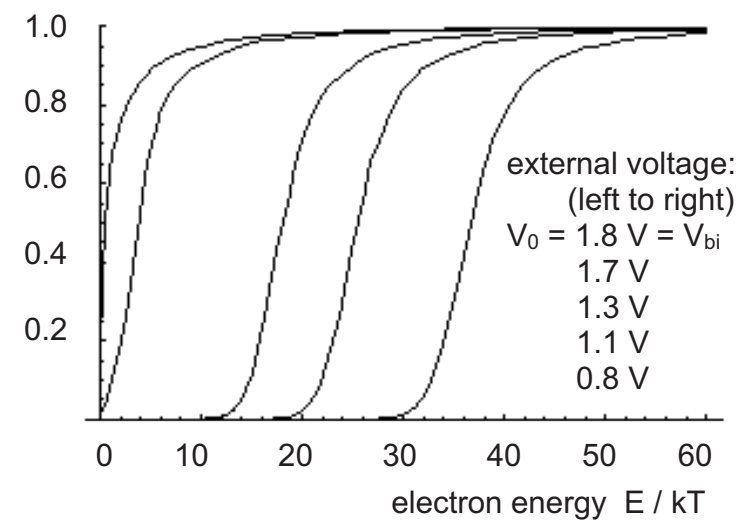

Fig. 2: The parabolic potential barrier at the abrupt $\mathrm{Np}^{+}$-heterojunction for various forward bias $V_{a}$ and the corresponding steady-state transmittance $T_{\mathrm{dc}}(E)$ 


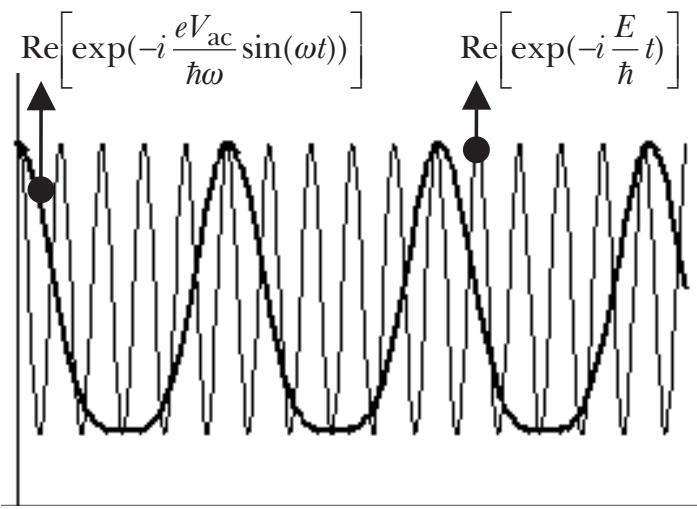

$(E / h) t$

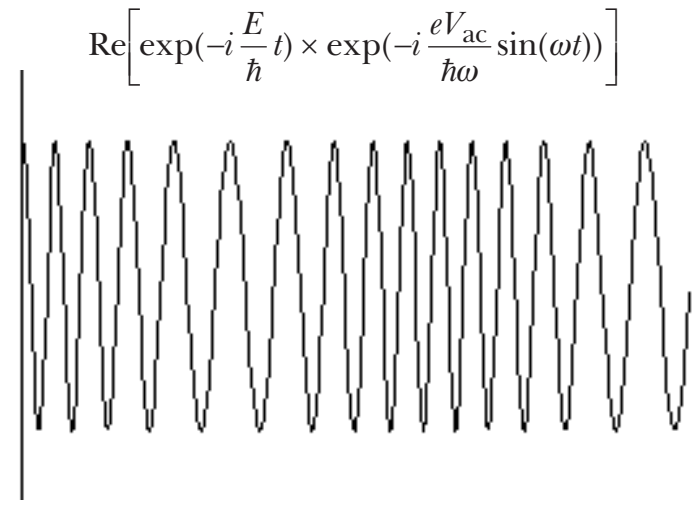

$(E / h) t$

Fig. 3: The real part of the electron wave function according to Eq. (10) for a rectangular barrier (height $200 \mathrm{meV}$, width $20 \mathrm{~nm}$ ), incident electron energy $50 \mathrm{meV}$, microwave signal frequency $1.2 \mathrm{THz}$

quantum $p \hbar \omega$. Its energy can be $E+p \hbar \omega, p=0, \pm 1, \pm 2, \ldots$, the \pm sign corresponds to the absorption/emission of energy quantum; $p=0$ means no emission or absorption.

As the electron energy can be $E+p \hbar \omega, p=0, \pm 1, \pm 2, \ldots$, the full electron wave function in regions A, B, C (see Fig. 1) should be the superposition of waves corresponding to these values of energy

$$
\begin{aligned}
& \psi_{\mathrm{A}}(x, t)= \exp \left(-i \frac{E}{\hbar} t\right) \times \\
& \times\left[\exp \left(i k_{0} x\right)+\sum_{n=-\infty}^{+\infty} r_{n} \exp (-i n \omega t) \exp \left(-i k_{n} x\right)\right], \\
& \psi_{\mathrm{B}}(x, t)= \exp \left(-i \frac{E}{\hbar} t\right) \times \\
& \times \sum_{n=-\infty}^{+\infty} \exp (-i n \omega t)\left\{\sum_{s=-\infty}^{+\infty}\left[a_{s} f(x)+b_{s} g(x)\right] J_{n-s}\left(\frac{e V_{\mathrm{ac}}}{\hbar \omega}\right)\right\}, \\
& \psi_{\mathrm{C}}(x, t)=\exp \left(-i \frac{E}{\hbar} t\right) \sum_{n=-\infty}^{+\infty} t_{n} \exp (-i n \omega t) \exp \left(i q_{n} x\right), \\
& E+n \hbar \omega=\hbar^{2} k_{n}^{2} / 2 m, \quad E+n \hbar \omega+\Phi_{\mathrm{B}}=\hbar^{2} p_{n}^{2} / 2 m .
\end{aligned}
$$

The function $\psi_{\mathrm{A}}$ is the superposition of the incident wave and the reflected waves with the reflectance amplitudes $r_{n}$, positive and negative values of $n$ correspond to the absorption and emission of energy quanta. The function $\psi_{\mathrm{C}}$ is the superposition of transmitted waves with the transmittance amplitudes $t_{n}$. The function $\psi_{\mathrm{B}}$ describes the electron motion across the barrier region (both the emission and the tunnelling).

The boundary conditions (4) should be now applied to the wave functions (12). Evaluating these relations and equating the terms at harmonics $\exp (-i n \omega t)$ we obtain a system of linear equations for the unknown coefficients $a_{n}, b_{n}, r_{n}, t_{n}$, $n=0, \pm 1, \pm 2, \ldots$. To calculate all these coefficients it would be necessary to solve an infinite set of linear algebraic equations. It is clear that the probability of emission or absorption of energy $n \hbar \omega$ decreases with increasing number $n$, thus the system could be terminated at some finite value of the indices $n, s$ in (12). The series expansion in (11) that results in the double summation in (12) is well known in the theory of frequency modulated signals in telecommunications and we can apply the result of signal theory: in the series expansion (11) it is sufficient to consider only the terms $n=0, \ldots, \pm N$, where $N$ is approximately equal to $e V_{\mathrm{ac}} / \hbar \omega$. If the high frequency signal is small it is sufficient to consider only $N=1$ or $N=2$, i.e. the generation of the first or the second harmonics or, in other words, the absorption or emission of one energy quantum $\hbar \omega$ or two energy quanta $2 \hbar \omega$. If the energy of the incident electron is $E$, the energy of the reflected or transmitted electron could be $E$ (unchanged, no absorption or emission), $E+\hbar \omega, E+2 \hbar \omega$ (absorption of one or two quanta), $E-\hbar \omega, E-2 \hbar \omega$ (emission of one or two quanta). For

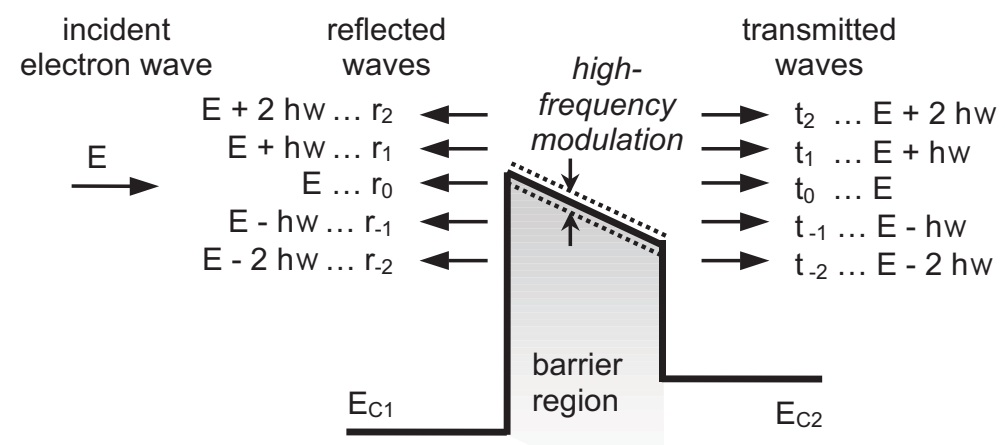

Fig. 4: High-frequency modulation of a potential barrier; $E$ is the incident electron energy, $\hbar \omega$ stands for the high-frequency quantum 

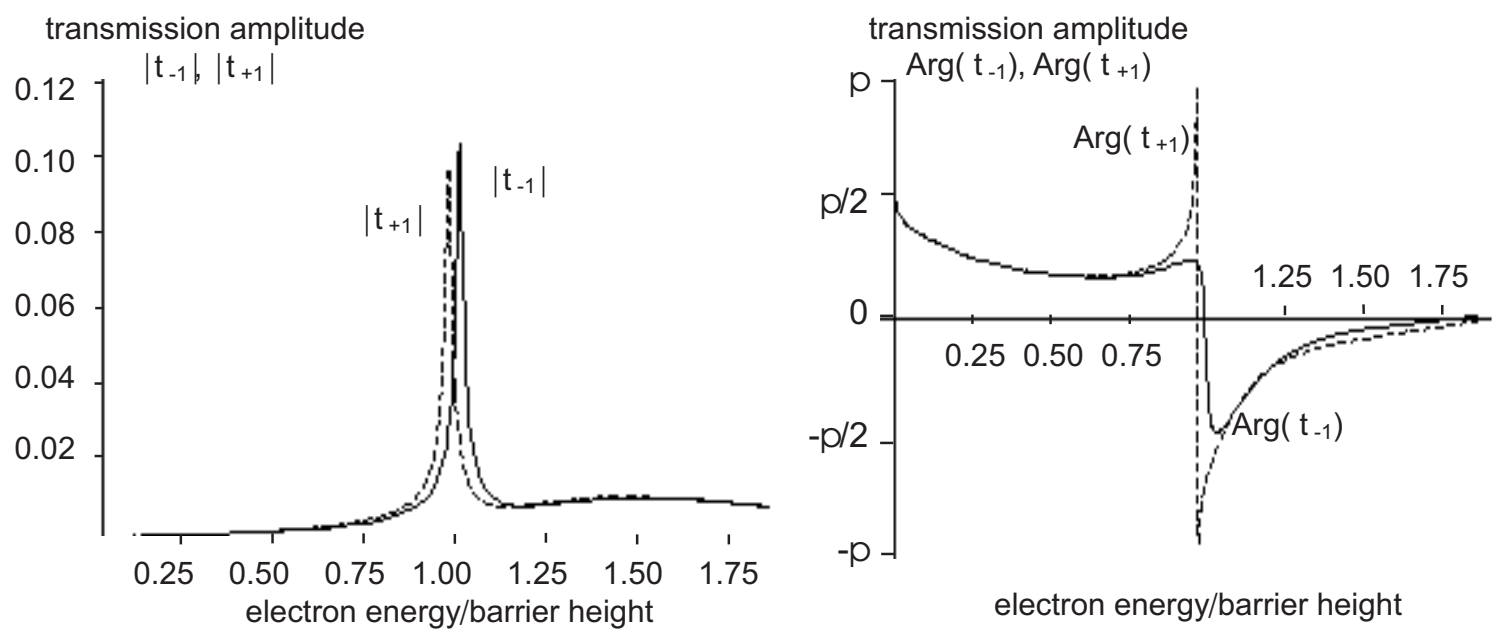

Fig. 5: Transmission amplitudes $t_{+1}, t_{-}$according to Eq. (13) for a rectangular barrier

$N=1$ (or 2) we obtain from Eq. (21) the system of 12 (or 20) linear equations for 12 (or 20) unknown coefficients; in general $8 N+4$ linear equations for $8 N+4$ unknown coefficients. Such system can be solved analytically in principle, but in practice numerical solution is used.

For the purpose of illustrating the above sketched theory it is useful to obtain some analytical results. We will consider the rectangular potential barrier in Fig. 1. If the amplitude of high frequency signal $V_{\mathrm{ac}}$ is small and the absorption or emission of only one quantum $\hbar \omega$ is considered the transmission amplitudes $t_{+1}$ (absorption, the electron energy in region $\mathrm{C}$ is $E+\hbar \omega$ ) and $t_{-1}$ (emission, the electron energy in region $\mathrm{C}$ is $E-\hbar \omega$ )

$$
\begin{aligned}
t_{ \pm 1} & = \pm \frac{e V_{\mathrm{ac}}}{2 \hbar \omega} t_{0} \times \\
& \times\left[\frac{\left(k_{0}+p_{0}\right) \cos \left(p_{0} x_{\mathrm{B}}\right)+i\left(p_{0}+\frac{k_{0} q_{0}}{p_{0}}\right) \sin \left(p_{0} x_{\mathrm{B}}\right)}{\left(k_{0}+p_{0}\right) \cos \left(p_{ \pm 1} x_{\mathrm{B}}\right)+i\left(p_{0}+\frac{k_{0} q_{0}}{p_{0}}\right) \sin \left(p_{ \pm 1} x_{\mathrm{B}}\right)}\right] .
\end{aligned}
$$

The transmission amplitude $t_{0}$ in (13) is given by the general formula (7), $k_{0}, q_{0}, p_{0}$ are the electron wave vectors defined in (1) and (3) and $p_{ \pm 1}=\sqrt{2 m\left(E \pm \hbar \omega-U_{\max }\right)} / \hbar$. Similarly as in (3) the quantities $p_{0}, p_{ \pm 1}$ are real for the electron emission over the barrier, and imaginary, thus $p_{0}=i \kappa_{0}$, $p_{ \pm 1}=i \kappa_{ \pm 1}$, for electron tunneling through the barrier. It can be seen in Fig. 3 that the modules $\left|t_{ \pm 1}\right|$ exhibit a strong resonant character at electron energy that corresponds to the barrier height.

\section{High frequency barrier transmittance}

If the transmission and reflection amplitudes are known, the wave functions (12) can be substituted to the general formulae (5) and the single electron quantum mechanical current densities of incident and transmitted electrons can be calculated. The high frequency barrier transmittance is defined as the ratio $\left|j_{\text {trans }}\right| /\left|j_{\text {inc }}\right|$ and can be found for each harmonic. If we adopt the approximation $k_{n} \approx k_{0}, q_{n} \approx q_{0}$ (as the electron energy is high compared with $\hbar \omega$ ) and restrict the calculation to the first harmonics, i.e. to the absorption or emission of one energy quantum, we obtain

$$
\begin{aligned}
& j_{\text {inc }}=e \frac{\hbar k_{0}}{m}, \\
& j_{\text {trans }}=e \frac{\hbar q_{0}}{m}\left[t_{0} t_{0}^{*}+\left(t_{0} t_{-1}^{*}+t_{1} t_{0}^{*}\right) e^{-i \omega t}+\left(t_{0}^{*} t_{-1}+t_{1}^{*} t_{0}\right) e^{i \omega t}\right] .
\end{aligned}
$$

We can see that $j_{\text {trans }}$ in (14) includes the dc component proportional to $t_{0} t_{0}^{*}$ (it is related to those electrons that pass the barrier region without absorption or emission of energy) and the ac component $\exp ( \pm i \omega t)$ related to electrons that emit or absorb one energy quantum in the barrier region. It is clear that the transmittance of the dc component is again given by (8), and it is not affected by the high frequency modulation. As usual in electronics, we use the goniometric functions $\sin (\omega t), \cos (\omega t)$ in (14) instead of complex functions $\exp ( \pm i \omega t)$ and denote $\theta_{1}=\arg \left(t_{0} t_{-1}^{*}+t_{1} t_{0}^{*}\right)$; the ac component then reads

$$
\begin{aligned}
j_{\text {trans }}^{(\omega)}= & 2 e \frac{\hbar q_{0}}{m}\left[\left|t_{0} t_{-1}^{*}+t_{1} t_{0}^{*}\right| \cos \theta_{1} \cdot \cos (\omega t)+\right. \\
& \left.+\left|t_{0} t_{-1}^{*}+t_{1} t_{0}^{*}\right| \sin \theta_{1} \cdot \sin (\omega t)\right]
\end{aligned}
$$

and the corresponding transmittances are

$$
\begin{aligned}
& T_{1 C}=2 \frac{q_{0}}{k_{0}}\left|t_{0} t_{-1}^{*}+t_{1} t_{0}^{*}\right| \cos \theta_{1}, \\
& T_{1 S}=2 \frac{q_{0}}{k_{0}}\left|t_{0} t_{-1}^{*}+t_{1} t_{0}^{*}\right| \sin \theta_{1} .
\end{aligned}
$$

More generally, if the $N$-quantum approximation is considered, the transmitted single electron quantum mechanical current density reads

$$
\begin{aligned}
& j_{\text {trans }}=\sum_{n=0}^{N} j_{\text {trans }}^{(n \omega)}, \\
& j_{\text {trans }}^{(n \omega)}=\frac{e \hbar q_{0}}{m}\left(T_{n} e^{-i n \omega t} T_{n}^{*} e^{i n \omega t}\right)
\end{aligned}
$$

and the transmittances $T_{n}$ in (17) for $N=3$ are given by 
$T_{0}=\left|t_{-3}\right|^{2}+\left|t_{-2}\right|^{2}+\left|t_{-1}\right|^{2}+\left|t_{0}\right|^{2}+\left|t_{1}\right|^{2}+\left|t_{2}\right|^{2}+\left|t_{3}\right|^{2}$

$T_{1}=t_{-3}^{*} t_{-2}+t_{-2}^{*} t_{-1}+t_{-1}^{*} t_{0}+t_{0}^{*} t_{1}+t_{1}^{*} t_{2}+t_{2}^{*} t_{3}$

$T_{2}=t_{-3}^{*} t_{-1}+t_{-2}^{*} t_{0}+t_{-1}^{*} t_{1}+t_{0}^{*} t_{2}+t_{1}^{*} t_{3}$

$T_{3}=t_{-3}^{*} t_{-0}+t_{-2}^{*} t_{1}+t_{-1}^{*} t_{2}+t_{0}^{*} t_{3}$

\section{Electrical parameters of quantum structure}

If the high frequency transmittances are known the electric current density $J^{(n \omega)}$ for each harmonic can be calculated by means of the well-known Tsu-Esaki formula $[1,4]$. We denote as $f(\varepsilon)$ the Fermi-Dirac function integrated over the parallel-to-interface wave vector components

$$
f(\varepsilon)=\ln \left[\frac{1+\exp \left(-\varepsilon+\frac{E_{C 1}-E_{F 1}}{k_{\mathrm{B}} T}\right)}{1+\exp \left(-\varepsilon+\frac{\left(E_{C 2}-E_{F 2}\right)-e V_{\mathrm{dc}}}{k_{\mathrm{B}} T}\right)}\right]
$$

with the dimensionless energy $\varepsilon=E / k_{\mathrm{B}} T$. The high frequency electric current harmonics can be written in the following way

$J^{(n \omega)}=A_{n} \cos (n \omega t)+B_{n} \sin (n \omega t)$

$A_{n}=\frac{e m k_{\mathrm{B}}^{2} T^{2}}{2 \pi^{2} \hbar^{3}} \int_{0}^{\infty} 2 \frac{q_{0}(\varepsilon)}{k_{0}(\varepsilon)}\left|T_{n}(\varepsilon)\right|\left|\cos \theta_{n}(\varepsilon)\right| f(\varepsilon) \mathrm{d} \varepsilon$

$B_{n}=\frac{e m k_{\mathrm{B}}^{2} T^{2}}{2 \pi^{2} \hbar^{3}} \int_{0}^{\infty} 2 \frac{q_{0}(\varepsilon)}{k_{0}(\varepsilon)}\left|T_{n}(\varepsilon)\right|\left|\sin \theta_{n}(\varepsilon)\right| f(\varepsilon) \mathrm{d} \varepsilon$

Observe that the origin of the higher order harmonics is related to the quantum character of electron transport in the barrier region rather than to the nonlinearity of current-voltage or capacitance-voltage characteristics. Thus, their existence is an intrinsic property of the quantum structure.

As our aim was to obtain the electrical parameters of the quantum structures, the relations (20) represent in fact the final result of the calculation. Using these formulae it is possible to find, e.g., the module of the higher order current harmonics and their phase shift with respect to the modulating signal (9) or the complex admittance and its real and imaginary part. All these quantities can be investigated as functions of the potential barrier profile (it is included in the barrier transmittance $T_{n}(\varepsilon)$ ), dc bias (included in $T_{n}(\varepsilon)$ and in $f(\varepsilon)$ ) or the angular frequency of the high frequency modulating signal (included again in $T_{n}(\varepsilon)$ ). The real and imaginary part of the complex admittance of the rectangular barrier for the first three harmonics as a function of frequency is shown in Fig. 6. The slope of the imaginary part of the admittance $\operatorname{Im}[y(\omega)]=\omega C$ implies that the capacitance is frequency independent.

\section{Conclusions}

The theory related to the transmittance of different types of potential barriers with dc bias and small high frequency ac signal in the terahertz frequency band was presented in this paper. We have followed the way from the hamiltonian and the time dependent Schrödinger equation to the electric current densities and complex admittance that can be measured in experiments. At such a high frequency the following effects could play an important role: the electron inside the barrier region can emit or absorb one or even more energy quanta $\hbar \omega$ where $\omega$ is the signal angular frequency. The electron wave function outside the barrier and consequently the electric current is a superposition of different harmonics $\exp (-i n \omega t)$. As we know from classical electronics, the generation of higher-order harmonics is due to the non-linearity of the current-voltage or capacitance-voltage characteristics, and it occurs only if the amplitude of the signal is sufficiently large. The origin of the higher-order harmonics at potential barriers is different: it is caused by the emission or absorption of one or more energy quantum and occurs even for a small signal; thus their generation is an intrinsic property of the single-barrier structure. The high frequency quantum effect on potential barriers represents an additional conductivity channel and contributes with a small parallel admittance to the electronic parameters of the structure.
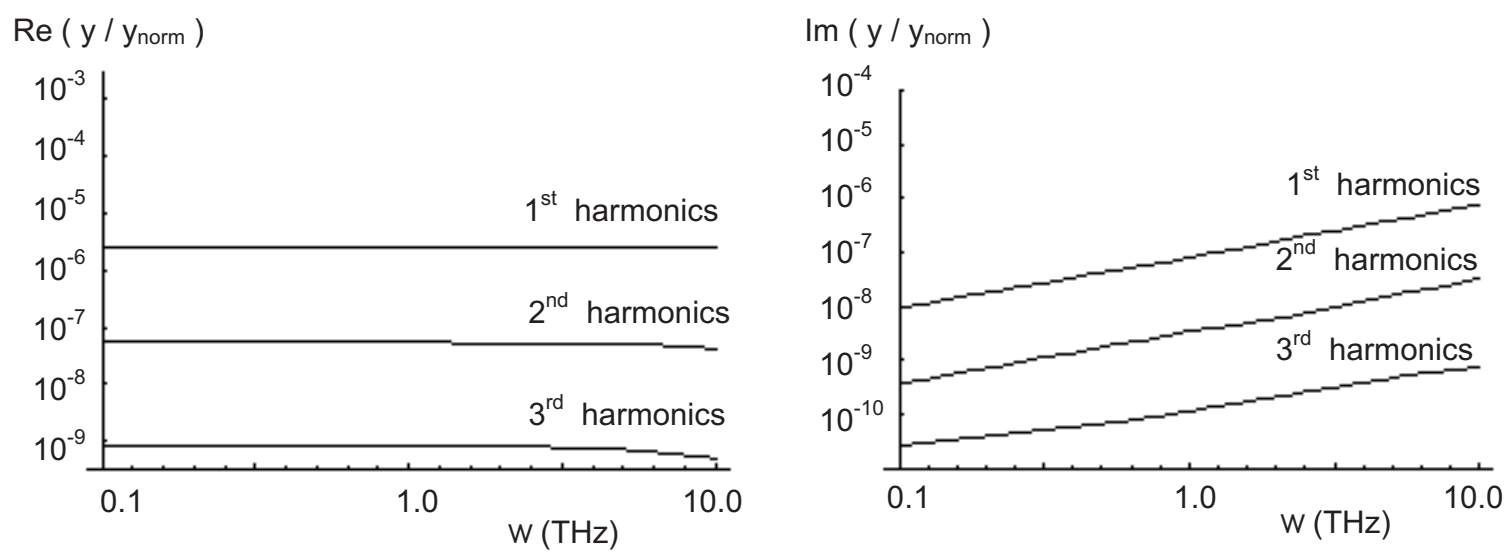

Fig. 6: The real and imaginary part of the complex admittance as functions of the modulation signal angular frequency for a rectangular potential barrier of height $300 \mathrm{meV}$ and width $16 \mathrm{~nm}$. The admittance is normalized by the quantity: $y_{\text {norm }}=\left(e m k_{B}^{2} T^{2} /\left(2 \pi^{2} \hbar^{3}\right) \times\left(e / k_{B} T\right)=2.72 \times 10^{11} \Omega^{-1} \mathrm{~m}^{-2}\right.$ 


\section{Acknowledgments}

This research has been supported by the Czech Ministry of Education in the framework of Research Plan MSM 262200022 MIKROSYT Microelectronic Systems and Technologies.

\section{References}

[1] Roblin P., Rohdin H.: High-speed heterostructure devices: from device concepts to circuit modeling. Cambridge University Press, 2002.

[2] Shore K. A.: "QC lasers may provide $\mathrm{THz}$ bandwith for communications". Laser Focus World, June 2002, p. 85-91.

[3] Bransden B. H., Loachaim C. J.: Introduction to quantum mechanics. Addison-Wesley Longman Ltd., 1989.

[4] Coon D. D., Liu H. C.: "Time-dependent quantum-well and finite-superlattice tunnelling". Journal Appl. Phys., Vol. 58 (1985), p. 2230-2235.

[5] Liu H. C.: "Analytical model of high-frequency resonant tunnelling: The first order ac current response". Phys. Rev. B, Vol. 43 (1991), p. 12538-12548.
[6] Truscott W. S.: "Wave functions in the presence of a time-dependent field: Exact solutions and their application to tunnelling". Phys. Rev. Lett. Vol. 70 (1993), p. 1900-1903.

[7] Fernando Ch. L., Frensley W. R.: "Intrinsic high-frequency characteristics of tunneling heterostructure devices". Phys. Rev. B, Vol. 52 (1995), p. 5092-5103.

[8] Tkachenko O. A., Tkachenko V. A., Baksheyev D. G.: "Multiple-quantum resonant reflection of ballistic electrons from a high-frequency potential step". Phys. Rev. $B$, Vol. 53 (1996), p. 4672-4675.

RNDr. Michal Horák, CSc.

e-mail: horakm@feec.vutbr.cz

Department of Microelectronics

Brno University of Technology

Faculty of Electrical Engineering and Communication

Údolní 53

60200 Brno, Czech Republic 